\title{
Nanoscale
}

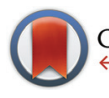

CrossMark

\& click for updates

Cite this: DOI: $10.1039 / \mathrm{c} 4 \mathrm{nr} 06513 a$

\section{Size and nanocrystallinity controlled gold nanocrystals: synthesis, electronic and mechanical properties $\dagger$}

\author{
N. Goubet, ${ }^{a, b}$ I. Tempra, ${ }^{c}$ J. Yang, $t^{a, b}$ G. Soavi, ${ }^{c} D$. Polli, ${ }^{c}$ G. Cerullo ${ }^{c}$ and \\ M. P. Pileni ${ }^{\star a, b, d}$
}

The influence of nanocrystallinity on the electronic and mechanical properties of metal nanoparticles is still poorly understood, due to the difficulty in synthesizing nanoparticles with a controlled internal structure. Here, we report on a new method for the selective synthesis of Au nanoparticles in either a singledomain or a polycrystalline phase maintaining the same chemical environment. We obtain quasi-spherical nanoparticles whose diameter is tunable from 6 to $13 \mathrm{~nm}$ with a resolution down to $\approx 0.5 \mathrm{~nm}$ and narrow size distribution (4-5\%). The availability of such high-quality samples allows the study of the impact of the particle size and nanocrystallinity on a number of parameters, such as plasmon dephasing time, electronphonon coupling, period and damping time of the radial breathing modes.

Received 5th November 2014 Accepted 8th January 2015

DOI: $10.1039 / c 4 n r 06513 a$

www.rsc.org/nanoscale
However, the obtained NPs are usually dispersible in water and have a size larger than $20 \mathrm{~nm} \cdot{ }^{11,12,17}$ Nonetheless, one parameter that is not yet well controlled is their nanocrystallinity, i.e. the amount of crystal defects inside the NP. Indeed, current syntheses are limited to the production of one form of nanocrystallinity, either single-domain or polycrystalline. ${ }^{12,18}$ The capability of synthesis of NPs with the desired nanocrystallinity with narrow size and shape distribution in an identical chemical environment (i.e. concentration and nature of reagents) is still lacking. ${ }^{22-27}$

Due to the limitations in synthesis control, only a few studies have been reported concerning the role of nanocrystallinity in the properties of metal NPs. ${ }^{22-24,27,28}$ This difficulty in synthesis is attributed to the low stacking-fault energy of gold $\left(\approx 40 \mathrm{~mJ} \mathrm{~m}{ }^{-2}\right)$, which implies that planar crystal defects in this material are easily formed. ${ }^{29}$ In NPs, the stacking faults often generate mirror planes that are named twins. This twinning process profoundly changes the internal structure of metal NPs and their overall shape. Indeed, the common polycrystalline forms of metal NPs are the multiply twinned particles (MTP), which are the decahedron and icosahedron. ${ }^{12,17,19,30}$ The former shape has 5 twins distributed on a quinary symmetry axis while the latter one has 30 twins distributed on 12 quinary symmetry axes. The single-domain shapes are well reproduced with the Wulff formula, which usually results in the truncated octahedron shape for fcc metals. ${ }^{31}$

It is known that the resistivity of metals decreases by increasing the amount of crystal defects, which act as scattering centers for the electrons. ${ }^{32}$ Consequently, the presence of twins should also modify the optical properties of gold NPs. 
Indeed, the localized surface plasmon resonance (LSPR) of NPs is a collective oscillation of their free electrons, which typically relaxes on the time scale of tens of femtoseconds. ${ }^{33-37}$ Following this initial ultrafast dephasing process, the photoexcited electrons lose their energy through several processes on different timescales, such as electron-electron ${ }^{6,38,39}$ $(\approx 100 \mathrm{fs})$ and electron-phonon ${ }^{27,40-42}(\approx 1 \mathrm{ps})$ collisions. At longer delays, the photon energy deposited into the NPs leads to lattice expansion and to the generation of coherent acoustic vibrations with a few-ps period. ${ }^{6,22-25,38,43-48}$ These lattice vibrations can be monitored either in the frequency domain (using low-frequency Raman spectroscopy), allowing the observation of the NP quadrupolar vibration modes, or in the time domain (through pump-probe spectroscopy), enabling the investigation of the NP radial breathing modes (RBMs). ${ }^{26,44,49-51}$ How these ultrafast relaxation processes in metal NPs are affected by nanocrystallinity is still a matter of debate.

Here, we report on a new method for the synthesis of $\mathrm{Au}$ NPs with different nanocrystallinity, size tunable between 6 and $13 \mathrm{~nm}$ and narrow size distributions (4-5\% rms diameter variation, as determined in the following). The quasi-spherical NPs are either in single-domain or polycrystalline phase, within the same chemical environment. The availability of such high quality samples allows us to study the effect of NP size and nanocrystallinity on their electronic and elastic properties. Electron scattering on crystal defects is studied through the LSPR linewidth while the ultrafast optical response of these colloidal solutions is obtained via pumpprobe experiments, which allow a detailed analysis of the NP electron-phonon coupling and coherent acoustic vibrations as a function of size and nanocrystallinity.

\section{Experimental}

\subsection{Synthesis of the seed gold NPs}

The gold NPs used as seeds for the size- and nanocrystallinitycontrolled growth are synthesized using a revisited method from the Stucky group. ${ }^{16}$ Briefly, $124 \mathrm{mg}$ of chlorotriphenylphosphine gold(I) is dissolved in $25 \mathrm{~mL}$ of toluene with $500 \mu \mathrm{L}$ of dodecanethiol. A solution containing $434 \mathrm{mg}$ of tert-butylamine borane complex in $5 \mathrm{~mL}$ of toluene is used to reduce the gold precursor. The reaction takes place at $100{ }^{\circ} \mathrm{C}$ and a dark red color rapidly appears when the reducing agent is added.

\subsection{Nanocrystallinity segregation}

The nanocrystallinity segregation was already reported in previous work. ${ }^{52,53}$ Before self-assembly process, the gold NP solution is washed with ethanol and the black powder is dispersed in toluene. In order to induce nanocrystallinity selection through self-assembly, one has to avoid solvent evaporation. To that end, the resulting colloidal solution is left destabilizing in a closed flask. After one week, a black precipitate, which is the nanocrystal superlattice, is recovered by centrifugation. Then, the solvent of a supernatant is replaced by hexane to obtain the polycrystalline seed solution. The black solid product is redispersed in hexane making the solution of single crystal seeds.

\subsection{Seeded growth with different nanocrystalline seeds}

The growth of the seeds is induced in a solution of Au-oleylamine complex, produced by mixing, at $35{ }^{\circ} \mathrm{C}, \mathrm{HAuCl}_{4}$ in pure oleylamine at $11 \mathrm{mmol} \mathrm{L}^{-1}$. After dissolution, the solution is yellow colored and freshly used. Then, $100 \mu \mathrm{L}$ of colloidal solutions at $1.4 \times 10^{-6} \mathrm{M}$ of either single-domain or polycrystalline $5 \mathrm{~nm}$ NPs are mixed with the Au-oleylamine complex solution and the volume is completed to $2 \mathrm{~mL}$ with toluene. The seeded growth takes place at $90{ }^{\circ} \mathrm{C}$. During 6 hours, Au-oleylamine complex is slowly reduced on the seeds. The resulting nanocrystal size can be controlled by adjusting the gold complex concentration. After reaction, the NPs are washed with $2 \mathrm{~mL}$ of ethanol and redispersed in chloroform.

\subsection{Electron microscopy}

The samples are prepared by depositing a drop of colloidal solution on a copper grid covered by an amorphous carbon film. The conventional TEM pictures are acquired with a JEOL 1011 at $100 \mathrm{kV}$. For the high-resolution experiments, a Jeol 2010 microscope is used at $200 \mathrm{kV}$. The size distribution and area of NPs are determined with the NIH ImageJ software.

\subsection{Wide-angle X-ray diffraction}

All the systems used to acquire X-ray diffraction patterns are under vacuum, from the emission of the copper anode to the detector. The detector is a curved photostimulable phosphor plate. After exposition, the film is scanned using a STORM 820 Molecular Dynamics scanner. The exposition is let running at least for 4 hours. The samples are made by evaporating the colloidal solution in a capillary tube with $100 \mu \mathrm{m}$ diameter. The resolution of the peak width due to the experimental set up is determined by using the $\{102\}$ and $\{201\}$ rays of $\mathrm{SiO}_{2}$ micrometric powder dispersed in oleylamine.

\subsection{Pump-probe experiment}

The experimental setup used for the pump-probe studies has been described elsewhere. ${ }^{54}$ It is based on a regeneratively amplified Ti:sapphire laser (Coherent, Libra) producing 100 fs, $4 \mathrm{~mJ}$ pulses at $800 \mathrm{~nm}$ wavelength and $1 \mathrm{kHz}$ repetition rate. A portion of this beam is frequency doubled to obtain the $400 \mathrm{~nm}$ pump pulses. Another fraction of the pulse is focused on a $2 \mathrm{~mm}$ thick sapphire plate to generate a broadband single-filament white-light continuum (WLC) that acts as a probe after passing through a delay line. The visible portion of the WLC, extending from $430 \mathrm{~nm}$ to $760 \mathrm{~nm}$, is overlapped with the pump beam on the sample. The transmitted/reflected light is dispersed on an optical multichannel analyzer equipped with fast electronics, allowing a single-shot recording of the probe spectrum at the full $1 \mathrm{kHz}$ repetition rate. By changing the pump-probe delay we record two-dimensional maps of the differential transmission $(\Delta T / T)$ signal as a func- 
tion of probe wavelength and delay. Our setup achieves, for each probe wavelength, a sensitivity down to $\approx 10^{-5}$. Temporal resolution (taken as full-width at half-maximum of pumpprobe cross-correlation) is $\approx 180$ fs over the entire probe spectrum.

\section{Results and discussion}

The synthesis starts by producing a mixture of single-domain and polycrystalline $5 \mathrm{~nm}$ NPs (Fig. 1a), ${ }^{26}$ revisiting the organometallic method developed by Stucky et $a l^{16}$ It has been demonstrated that nanocrystallinity segregation occurs by allowing such a colloidal solution under toluene saturation over 7 days. Indeed, low frequency Raman spectra show that the polycrystalline NPs are still dispersed in the solution whereas single-domain self-assemble into a 3D superlattice during the destabilization of the colloidal solution (Fig. S1†). ${ }^{52,53}$ The resulting superlattices exhibit orientational ordering which implies an atomic alignment between the single-domain NPs. This crystallographic register explains the stronger interactions between single-domain NPs compared to the polycrystalline ones, induced by nanocrystal facet-to-facet geometry. Thanks to this crystallinity segregation process, we are able to selectively synthesise $5 \mathrm{~nm}$ single-domain and polycrystalline NPs, both dispersed in hexane (Fig. 1b, 1f). Such
NPs are used as seeds to selectively grow single-domain or polycrystalline NPs differing by their sizes (Fig. 1a). The growth of the seeds is induced in a solution of Au-oleylamine complex. The gradual $\mathrm{Au}$ input on the seed surface avoids any nucleation of new NPs. In this way, the obtained polydispersity of NP diameters is low (3-7\%), as shown in Fig. 1b-i and in ESI (Fig. S2 and S3 $\uparrow$ ). Moreover, the size distributions display Gaussian profiles, thus confirming that the growth of the seeds is homogeneous and it cannot be attributed to sintering or Ostwald ripening of NPs, which usually leads to a lognormal distribution (see ESI, Fig. S2 and S3†). ${ }^{55}$ The mean NP diameter is controlled by the Au complex concentration in the reaction solution, determined by the volume of Au-oleylamine input. The determination of the nanocrystallinity purity is impossible with the various techniques used in this study since the XRD measurements provide an average response of the nanocrystals. Moreover, one has to take into account that crystallinity segregation can occur during the monolayer formation on the TEM grid and therefore this effect will distort the purity percentage due to the local probing area. This makes the determination of the purity percentage impossible. However, the crystallinity purity of the seeds has been investigated by low frequency Raman spectroscopy (Fig. S1 $\dagger$ ). ${ }^{52}$ As shown in the ESI, $\uparrow$ the signature of polycrystalline population is quite pure and free from single crystals and vice versa. Then, we can conclude that the remaining crystallinity impurities on
Step 1

Synthesis of nanocrystallinity mixture

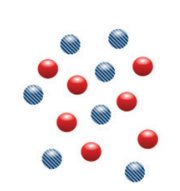

$\mathrm{b}$

$\mathrm{f}$

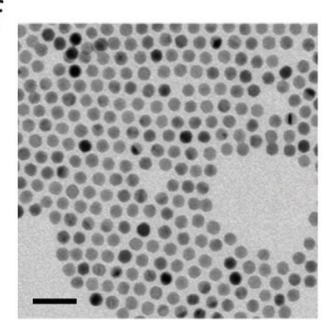

Step 2

Crystallinity segregation

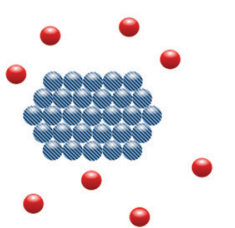

d

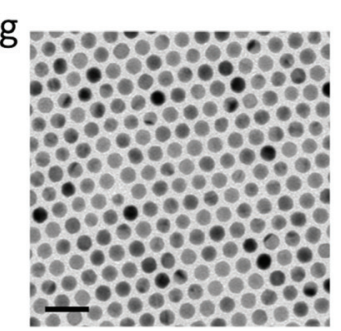

$h$

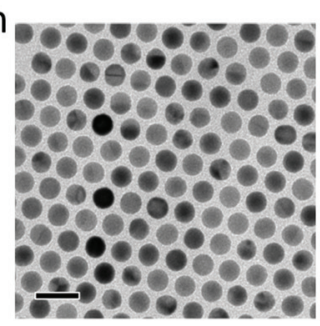

a
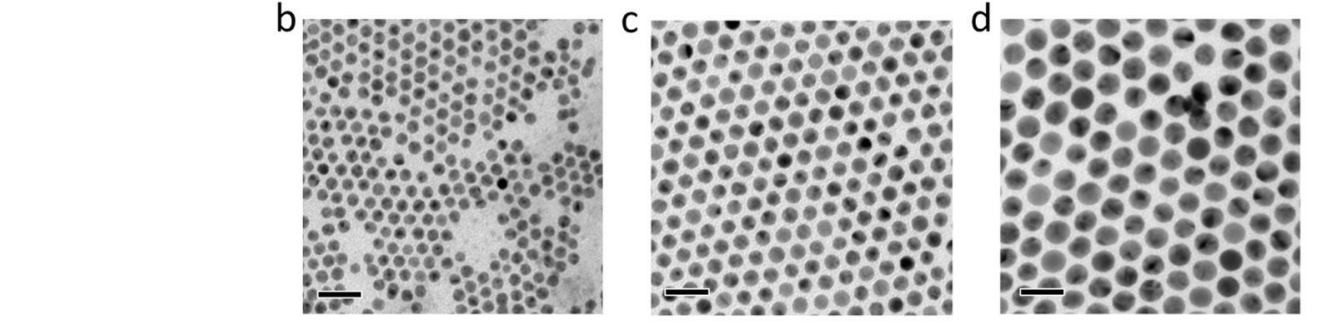

Step 3

Seeded growth

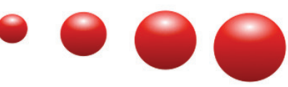

*
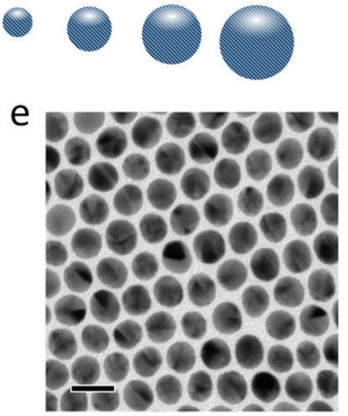

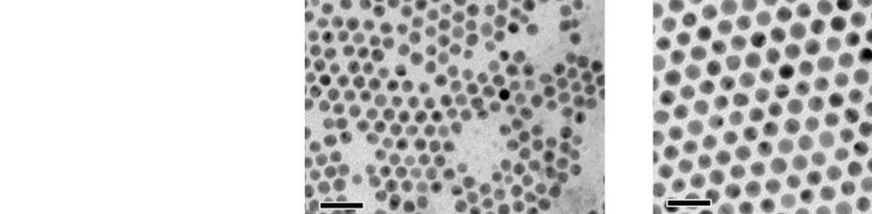

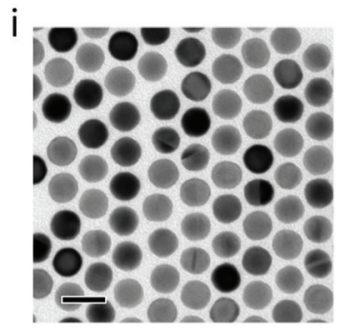

Fig. 1 (a) A schematic image showing the formation of gold NPs with tunable size and nanocrystallinity, the polycrystalline NPs are represented in red and single-domain in blue with straight lines. (b-e) TEM images of NPs synthesized using polycrystalline seeds, (b) $5.6 \mathrm{~nm}$; (c) $6.8 \mathrm{~nm}$; (d) $9.6 \mathrm{~nm}$ and (e) $12.7 \mathrm{~nm}$. (f-i) TEM images of NPs synthesised using single-domain seeds, (f) $5.9 \mathrm{~nm}$; (g) $6.7 \mathrm{~nm}$; (h) $9.6 \mathrm{~nm}$ and (i) $13.2 \mathrm{~nm}$. The scale bars are $20 \mathrm{~nm}$. 


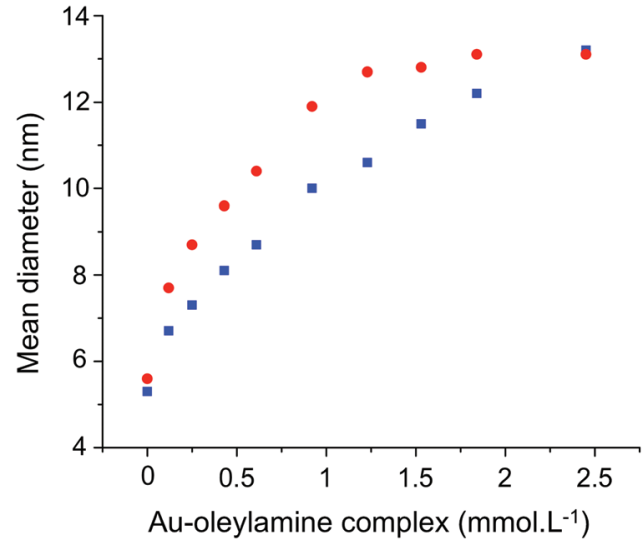

Fig. 2 Typical mean diameter of single-domain (blue squares) and polycrystalline (red circles) NPs as a function of gold complex concentration during the seeded growth.

the TEM pictures should be negligible. As shown in Fig. 2, the seeded growth is slightly faster in the case of polycrystalline NPs compared to single-domain ones. By finely tuning the Auoleylamine concentration, the control of mean diameter can be reduced to $0.5 \mathrm{~nm}$. With this method we obtain size tunability between $5 \mathrm{~nm}$ (seeds) and $13 \mathrm{~nm}$ for both singledomain and polycrystalline NPs.

\subsection{Nanocrystallinity}

The nanocrystallinity of Au NPs was investigated by X-ray diffraction (XRD) and high-resolution transmission electron microscopy (HRTEM). Fig. 3a shows typical diffraction profiles of $\{111\}$ and $\{200\}$ Au lattice planes. The blue and red curves are obtained from $13.2 \mathrm{~nm}$ and $13.1 \mathrm{~nm}$ NPs synthesized from single-domain and polycrystalline seeds, respectively. The diffraction profiles of the other NPs with a different diameter are shown in the ESI (Fig. S4 and S5†). The crystallite size, $D_{\text {XRD }}$, is determined from the Scherrer formula ${ }^{56}$ using the broadening of the peak extracted from a pseudo-Voigt fit. For NPs produced using single-domain seeds, Fig. 3b shows a good agreement between the $D_{\mathrm{XRD}}$ determined from both $\{111\}$ and $\{200\}$ reflections and the NP diameter determined by TEM $\left(D_{\text {TEM }}\right)$. This indicates that the overall populations are single-domain without any anisotropy in the crystallite shape (quasi-sphere). The HRTEM images (Fig. 3c, 3f) show the lattice fringes of single domain NP and confirm the nearly spherical NP shape with small flat facets. In the case of NPs synthesized with polycrystalline seeds, the crystallite size is smaller on the $\langle 200\rangle$ directions compared to the $\langle 111\rangle$, and both are smaller than $D_{\text {TEM}}$. This anisotropic shape of the crystallites is confirmed by the HRTEM images, where two main MTPs with tetrahedral sub-units are observed: decahedron (Fig. 3d, 3g) and icosahedron (Fig. 3e). As with single-domain a

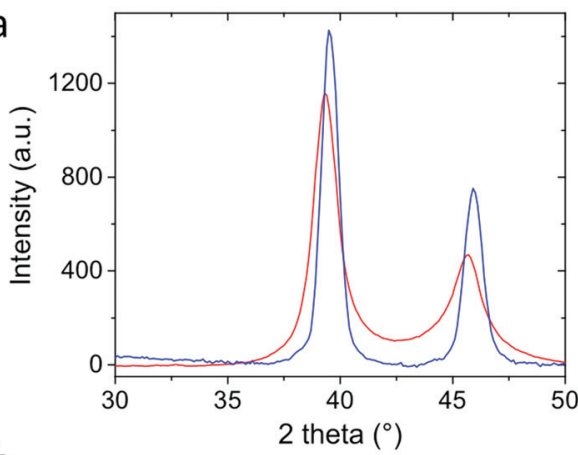

$b$

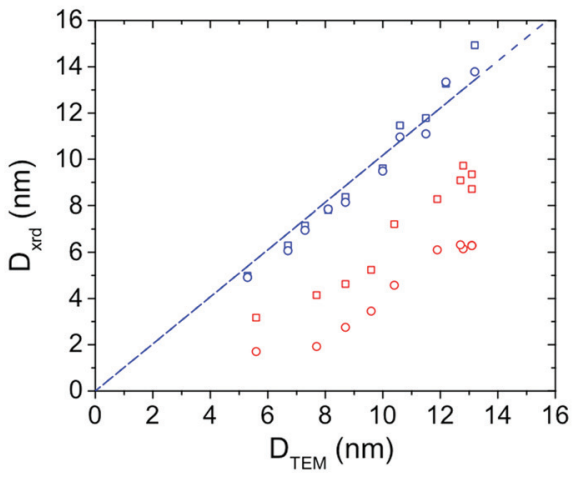

C
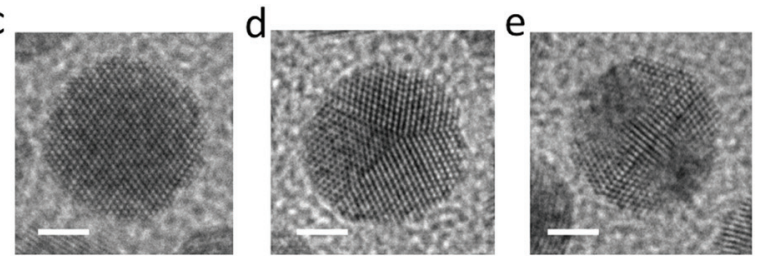

f
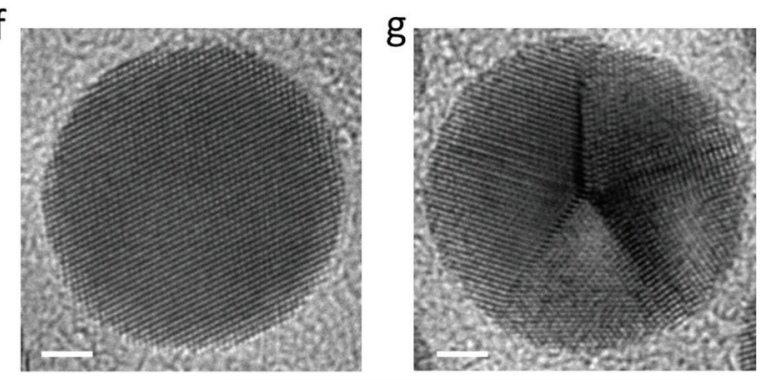

$\mathrm{h}$

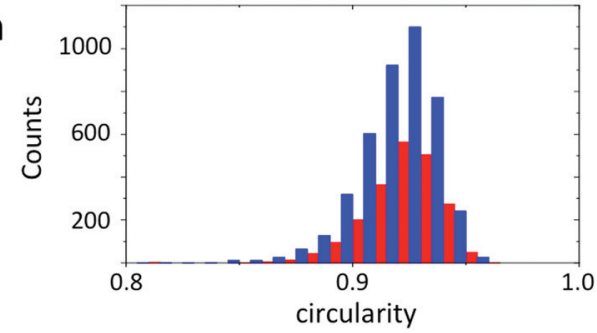

Fig. 3 (a) XRD patterns of single-domain (blue) and polycrystalline NPs (red) showing the two first Bragg peaks $\{111\}$ and $\{200\}$. (b) Crystallite size obtained by XRD from the $\{111\}$ reflection (square) and $\{200\}$ reflection (circle) against the NP diameter obtained by TEM for single-domain (blue) and polycrystalline NPs (red). Each symbol corresponds to a different synthesis run (c-e) HRTEM images of $6 \mathrm{~nm}$ NPs and ( $\mathrm{f}-\mathrm{g}$ ) $12 \mathrm{~nm}$ NPs with (c, f) single crystal, (d, g) decahedra and (e) icosahedron, all scale bars are $2 \mathrm{~nm}$. (h) Circularity distribution of $10.6 \mathrm{~nm}$ single-domain (blue) and polycrystalline NPs (red). 
NPs, the edges of the polycrystals are round (Fig. $3 g$ ). The oxidative etching of oleylamine in the presence of $\mathrm{O}_{2}$ on Au NPs is well known. ${ }^{57}$ This etching process is reported to select single-domain instead of polycrystalline NPs via dissolution of Au preferably on crystal defects. Here, the solutions are already with selected nanocrystallinity and no selective etching is observed. Whatever their nanocrystallinity, the NPs are etched on their vertices and edges during their growth. This results in a nearly spherical shape with distribution of vicinal surfaces between their small facets. In order to characterize a possible shape variance between single-domain and polycrystalline NPs, the circularity " $c$ " of the Au NP is defined as:

$$
c=\frac{A_{\mathrm{NP}}}{A_{\mathrm{disc}}}
$$

with $A_{\mathrm{NP}}$ the area of the NP projection on TEM pictures and $A_{\text {disc }}$ the area corresponding to the perfect circumscribed disc. The larger diameter of the NP projection, also called Feret diameter, is used to determine $A$. Fig. 3 h shows the circularity distribution histograms of both single-domain (blue) and polycrystalline (red) $6.6 \mathrm{~nm}$ NPs: they are nearly fully overlapped. From this we conclude that both single-domain and polycrystalline NPs can be seen as quasi-spheres. Similar circularity distributions for NPs with different diameters are shown in the ESI (Fig. S6 $\dagger$ ). By fitting the XRD pattern with a pseudoVoigt function, it is possible to extract the Lorentz factor and determine if the peak broadening is mainly Lorentzian or Gaussian (see Table S1 in the ESI $\dagger$ ). The main contribution for polycrystalline NPs is clearly Lorentzian indicating a larger crystallite size distribution due to the different tetrahedral subunit size of icosahedron and decahedron. In the case of singledomain NPs, the Gaussian profile reveals the narrow crystallite size (i.e. NP size) distribution. Hence, starting from $\mathrm{Au}$ seeds with controlled nanocrystallinity, it is possible to synthesize nearly spherical NPs with a tunable diameter, a narrow size distribution and with the same chemical environment.

\subsection{Localized surface plasmon resonance}

The NPs coated with dodecanethiol are dispersed in chloroform after synthesis. We have recorded the linear absorption spectra of single-domain and polycrystalline NPs with different sizes. Fig. 4a shows representative examples for NPs with $6.6 \mathrm{~nm}$ diameter: the spectra are dominated by the LSPR absorption band at $527 \mathrm{~nm}$. As already observed for $5 \mathrm{~nm}$ nanoparticles, ${ }^{23}$ for any size in the 6 to $13 \mathrm{~nm}$ range, the LSPR band of polycrystalline NPs is characterized by a weaker intensity and larger linewidth compared to single-domain NPs having the same average size. Inset of Fig. 4a confirms that in the size range studied here, according to the well-known Fröhlich formula, the LSPR position does not depend on the NP diameter, so that the influence of the narrow size distribution on the broadening of the LSPR band is negligible. The optical properties of Au NPs larger than $5 \mathrm{~nm}$ can be well represented with a classical approach, ${ }^{58}$ in which the LSPR

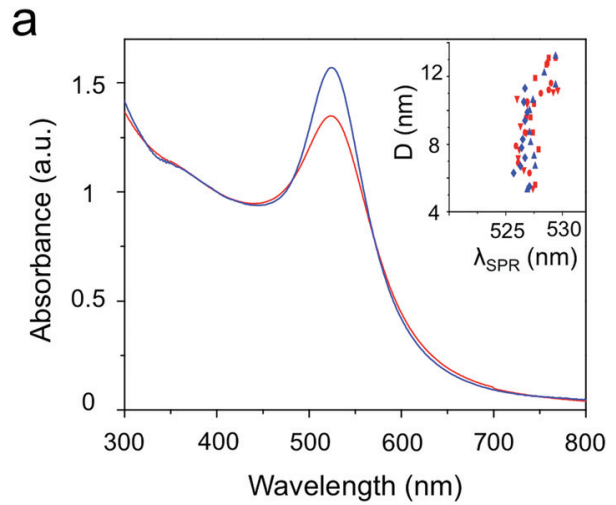

b

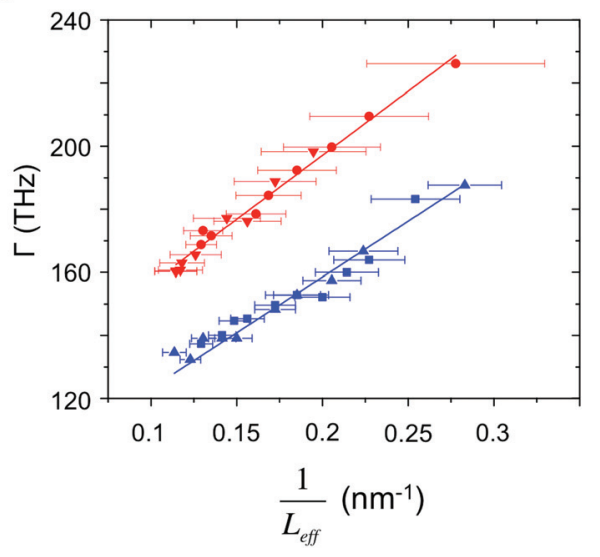

Fig. 4 (a) Typical localized surface plasmon resonance (LSPR) for an ensemble of single-domain (blue) and polycrystalline (red) NPs with $6.6 \mathrm{~nm}$ as diameter, (inset) dependence of the LSPR position on the nanocrystal size. (b) LSPR linewidth versus the inverse of the radius for single-domain (blue squares) and polycrystalline (red circles) NPs; each symbol corresponds to a different synthesis run.

linewidth is related to the electronic scattering rate and given by:

$$
\Gamma=\Gamma_{0}+\frac{A v_{\mathrm{f}}}{R}
$$

where $\Gamma_{0}, A, v_{\mathrm{f}}$ and $R$ are the intrinsic electron scattering rate, a constant dependent on the dielectric constant of the medium, the Fermi velocity of electrons and the NP radius, respectively. From the circularity data shown above, to a first approximation, we can assume that the NPs are spherical. The intrinsic electron damping constant is represented by the different electron collision events which are defined by the Matthiessen law $^{9}$ and the absorption due to the interband transition. ${ }^{38}$ Indeed, since the LSPR of Au NPs partly overlaps with the interband transition due to its short-wavelength threshold $(\approx 540 \mathrm{~nm})$, one has to take it into account in the following description:

$$
\Gamma_{0}=\frac{1}{\tau_{\mathrm{e}-\mathrm{ph}}}+\frac{1}{\tau_{\mathrm{e}-\mathrm{e}}}+\frac{1}{\tau_{\text {defect }}}+\frac{\omega_{\mathrm{LSPR}}^{3}}{\omega_{\mathrm{p}}{ }^{2}} \varepsilon_{2}^{\mathrm{ib}}
$$

where $\tau_{\mathrm{e}-\mathrm{ph}}, \tau_{\mathrm{e}-\mathrm{e}}, \tau_{\mathrm{defect}}$ are the damping times due to the scattering of the electrons on phonons, electrons and lattice 
defects (e.g. point defects, dislocations and grain boundaries), respectively. The last term describes the absorption due to interband transition where $\omega_{\mathrm{LSPR}}$ is the LSPR frequency, $\omega_{\mathrm{p}}$ is the plasma frequency of the metal and $\varepsilon_{2}^{\text {ib }}$ is the imaginary part of the interband contribution of bound electrons to the dielectric function. ${ }^{38}$ Note that due to the size dependences of $\tau_{\mathrm{e}-\mathrm{ph}}$ and $\tau_{\mathrm{e}-\mathrm{e}},{ }^{38,40} \Gamma_{0}$ is different from the bulk value. However, since the $\tau_{\mathrm{e}-\mathrm{ph}}$ and $\tau_{\mathrm{e}-\mathrm{e}}$ size dependences mostly concern NPs with diameter below $5 \mathrm{~nm}$, it can be neglected in our case. The experimental LSPR absorption band is fitted with the superposition of a Lorentzian function and a linear decay that takes into account the absorption due to interband transitions (see ESI, Fig. S7†). From such analysis we extract the LSPR linewidth $(\Gamma)$ of our samples. Fig. 4b shows the relationship between $\Gamma$ and the inverse of the NP radius for single-domain (blue) and polycrystalline (red) NPs. It is worth noting that these results are reproducible from one set of synthesis to the other. A linear fit allows us to determine the values of the intrinsic scattering rate $\Gamma_{0}$ and of the constant $A$, which are $(11.3 \mathrm{fs})^{-1}, 0.19$ and $(8.6 \mathrm{fs})^{-1}, 0.22$ for single-domain and polycrystalline NPs, respectively. The difference in the linewidths cannot be attributed to the chemical interface damping, since the NPs are synthesized in the same chemical environment. The similarity of the constant $A$ for both nanocrystallinities confirms that the chemical environment and consequently the dielectric constant of the medium remains unchanged, suggesting comparable electron-surface scattering processes. Since $\omega_{\text {LSPR }}$ does not depend on the nanocrystal size (inset Fig. 4a), the absorption due to the interband transition is similar for single-domain and polycrystalline NPs. Then, the observed difference in $\Gamma_{0}\left((11.3 \mathrm{fs})^{-1}\right.$ and $\left.(8.6 \mathrm{fs})^{-1}\right)$ between single-domain and polycrystalline NPs is attributed to crystal defects increasing the scattering rate, as shown in eqn (3). In particular it has been shown that the plasmon linewidth of polycrystalline gold NPs is strongly affected by the grain size. ${ }^{59}$ We conclude that the collection of polycrystalline NPs have intrinsic dephasing rates around $20 \%$ higher than singledomain NPs due to the electron scattering on their crystal defects. This result reveals the important and often neglected role of twins in determining the LSPR linewidth. Indeed, the main parameter that is used to calculate the NP optical response obtained with single-particle spectroscopy is the surface scattering parameter, $A \cdot{ }^{35,58}$ However, the values of this parameter are often different from one NP to the other while the crystal defects can also contribute to the LSPR broadening and have to be taken into account.

\subsection{Femtosecond broadband pump-probe measurements}

The availability of high-quality size-controlled samples, with either poly or single-crystalline phase, allows us to study the effect of size and nanocrystallinity on the electronic and vibrational dynamics of gold NPs. We performed pump-probe measurements of the NPs dissolved in chloroform in a $1 \mathrm{~mm}$ optical-path cuvette. The sample was excited at $400 \mathrm{~nm}$ and the differential transmission $(\Delta T / T)$ signal was measured over the entire visible spectral region using a white-light supercon-

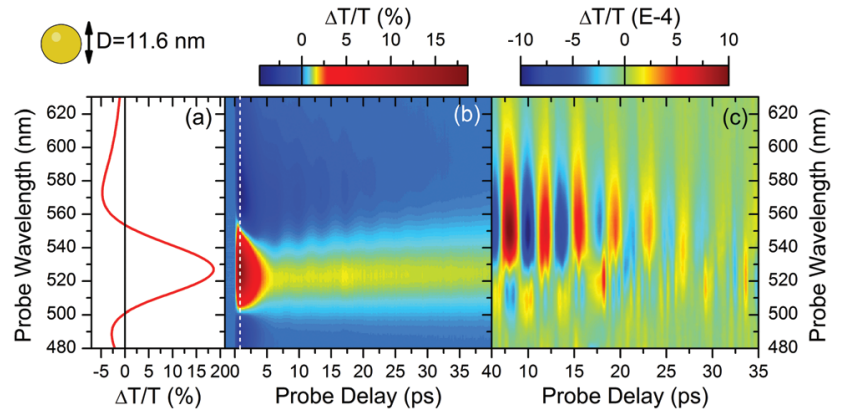

Fig. 5 Broadband femtosecond pump-probe spectroscopy for a single-crystal sphere of $11.6 \mathrm{~nm}$ diameter excited at $400 \mathrm{~nm}$. (a) Spectrum at 800 fs pump-probe delay showing the typical "second derivative" shape due to broadening of the plasmonic resonance. (b) Twodimensional $\Delta T / T$ map showing the signal as a function of pump-probe delay and probe wavelength. The vertical cut (dotted line) at 800 fs shows the position of the transient spectrum depicted in panel (a). (c) Map of the coherent oscillations on the two sides of the plasmonic resonance obtained by subtracting the slowly varying exponential decays to the map in panel (b).

tinuum probe pulse entirely covering the LSPR of the different nano-spheres. ${ }^{46}$ Fig. 5 shows typical results for $11.6 \mathrm{~nm}$ diameter single-domain NPs. The two-dimensional $\Delta T / T$ map as a function of the probe wavelength and pump-probe delay is presented in panel (b), while panel (a) depicts a vertical cut, namely a $\Delta T / T$ spectrum for a fixed pump-probe delay of 800 fs. Panel (b) also highlights the typical shape of Au NPs transient spectra after photoexcitation: ${ }^{48}$ the $400 \mathrm{~nm}$ pump pulse mainly promotes electrons from the $5 \mathrm{~d}$ to the $6 \mathrm{sp}$ Au bands and creates a non-thermal electronic distribution that recovers to the initial equilibrium conditions through a sequence of processes, such as electron-electron and electron-phonon scattering and heat dissipation to the surroundings. ${ }^{6}$ The increase in the electronic temperature leads to a broadening of the LSPR due to an increase of the imaginary part of the Au dielectric constant. This broadening is responsible for the "second derivative" shape observed in Fig. 5(a) and peaked at approximately $525 \mathrm{~nm}$, where the LSPR for small spherical Au NPs (whose extinction coefficient is dominated by absorption rather than scattering) occurs. ${ }^{47}$ The electron-phonon relaxation process discussed is also responsible for an energy flow from the electrons to the lattice and, as a consequence, it drives an impulsive heating and expansion of the NPs. Because the time scale for heating ( $\approx 1$ picosecond) is typically faster than the period of the fundamental RBM in small nanoparticles, this expansion impulsively launches coherent oscillations that are coupled to the optical response of the sample through a periodical shift in the position of the LSPR peak. ${ }^{6}$ In the case of Au spherical NPs they arise from periodical variations of the volume and, consequently, of the carrier density which directly affects the plasma frequency. An example of the measured coherent oscillations in the $11.6 \mathrm{~nm}$ diameter single-domain NPs is highlighted in Fig. 5, where in panel (c) we plot a close up of the recorded $\Delta T / T$ signal (shown in panel b), for delays longer than 6 ps (to remove the initial fast 
thermalization process). We isolate the oscillatory component of the signal by subtracting the slowly varying relaxation dynamics for each probe wavelength. It is worth noting that the amplitude of the oscillation is larger at longer wavelengths $(540-570 \mathrm{~nm})$ with respect to the plasmonic peak $(\approx 525 \mathrm{~nm})$ and a $\pi$ phase shift occurs for wavelengths at different sides of the LSPR, ${ }^{46}$ as expected from oscillations arising from a periodic shift of an optical resonance. In the following, we will first investigate the electron-phonon scattering dynamics, which occur during the first $\approx 5$ ps after excitation, and then we will focus on the analysis of the coherent oscillations on the longer timescale.

\subsection{Electron-phonon scattering}

It is known that the time constant for the electron-phonon scattering increases linearly with the excitation fluence; ${ }^{41}$ this can be explained in the framework of the two-temperature model given that a higher pump intensity creates a higher initial electronic temperature that is directly reflected in the relaxation time constant. ${ }^{47}$ Indeed, at low pump power, the electron-phonon scattering time constant is given by

$$
\tau_{\mathrm{e}-\mathrm{ph}}=\gamma\left(T_{0}+\Delta T\right) / g
$$

where $\gamma$ is the electron heat capacity constant, $g$ is the electron-phonon coupling constant, $T_{0}$ and $\Delta T$ are the ambient temperature and the pump-induced temperature increase, respectively. Among the parameters that can affect the electron-phonon scattering rate, the nanocrystallinity of the sample is believed to play a fundamental role but still experiments in this direction are controversial. ${ }^{27,28}$ To clarify this issue, we performed fluence-dependent measurement on $\mathrm{Au}$ NPs of the same size that only differ in terms of their nanocrystallinity. In Fig. 6(a) we show the measured (data points) $\Delta T / T$ dynamics at $525 \mathrm{~nm}$ (i.e. the peak of the bleaching signal) at three different pump fluences (normalized to the low-fluence signal) for the single-domain NPs with $10.6 \mathrm{~nm}$ diameter. The fits (solid lines) are performed considering the instrumental response function of our system and the contribution of three exponential processes, representing the electron-electron, electron-phonon and phonon-phonon scattering processes. The electron-electron and phonon-phonon time constants were fixed while the electron-phonon decay was the only free parameter. In Fig. 6(b) we plotted the extracted electron-phonon time constant $\tau_{\mathrm{e}-\mathrm{ph}}$ as a function of the pump fluence for single-domain (blue squares) and polycrystalline (red circles) NPs. The two samples are diluted so as to have the same steady-state absorption at $400 \mathrm{~nm}$, in order to guarantee that the absorbed energy is the same at any given pump fluence. Interestingly, we observe that the nanocrystallinity does not affect the electron-phonon scattering dynamics, as the two datasets are overlapping. By extending the linear fit of the data (black solid line in Fig. 6(b)) to the intercept with the vertical axis, we can extrapolate an electron-phonon time constant at zero-power excitation of approximately 625 fs. This value, in good agreement with other measurements on $\mathrm{Au}$ nanoparticles, ${ }^{41,47}$ corresponds to the value of bulk $\mathrm{Au}^{60}$ and further confirms the quality of our results. Very similar results were obtained for another couple of samples consisting of single-domain and polycrystalline NPs with $7.7 \mathrm{~nm}$ diameter.

\subsection{Radial breathing mode oscillations}

Let us now turn to the study of the coherent oscillations for $\mathrm{Au}$ NPs differing by size and nanocrystallinity. In the case of spherical NPs, the vibrational modes can be accurately described as those of an elastically isotropic homogeneous sphere $^{61}$ and time-domain experiments mainly probe their fundamental RBM, due to isotropy of the thermal exci-
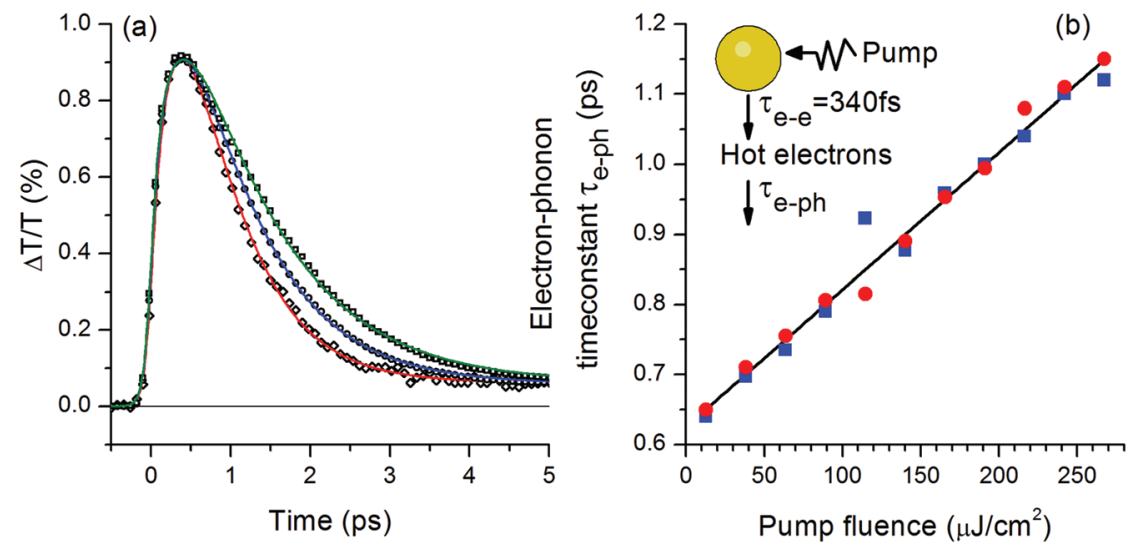

Fig. 6 Effect of the nanocrystallinity on the electron-phonon scattering. (a) $\Delta T / T$ dynamics (data points) for single-domain NPs with $10.6 \mathrm{~nm}$ diameter excited at $400 \mathrm{~nm}$ and corresponding fitting (solid lines) obtained considering $180 \mathrm{fs}$ temporal resolution, a fixed electron-electron scattering time constant of $340 \mathrm{fs}$ and an electron-phonon scattering time-constant as a free parameter. Pump fluences: $12.7 \mu \mathrm{J} \mathrm{cm}{ }^{-2}$ (diamonds and red curve), $140 \mu \mathrm{J} \mathrm{cm}^{-2}$ (circles and blue curve, scaled down by 8.7 times to normalize the trace to the low-fluence data) and $267 \mu \mathrm{J} \mathrm{cm}{ }^{-2}$ (squares and green curve, scaled down by 15.9 times). The electron-phonon scattering time-constant is kept as a free parameter. (b) Extracted electron-phonon scattering time constant as a function of pump fluence for both single-domain (blue squares) and polycrystalline (red circles) NPs with $10.6 \mathrm{~nm}$ diameter; solid black line is a linear fit to the data (intercept at $\tau_{\mathrm{e}-\mathrm{ph}}^{0}=625 \mathrm{fs}$; slope $1.96 \mathrm{fs} \mathrm{cm}^{2} \mu \mathrm{J}^{-1}$ ). 

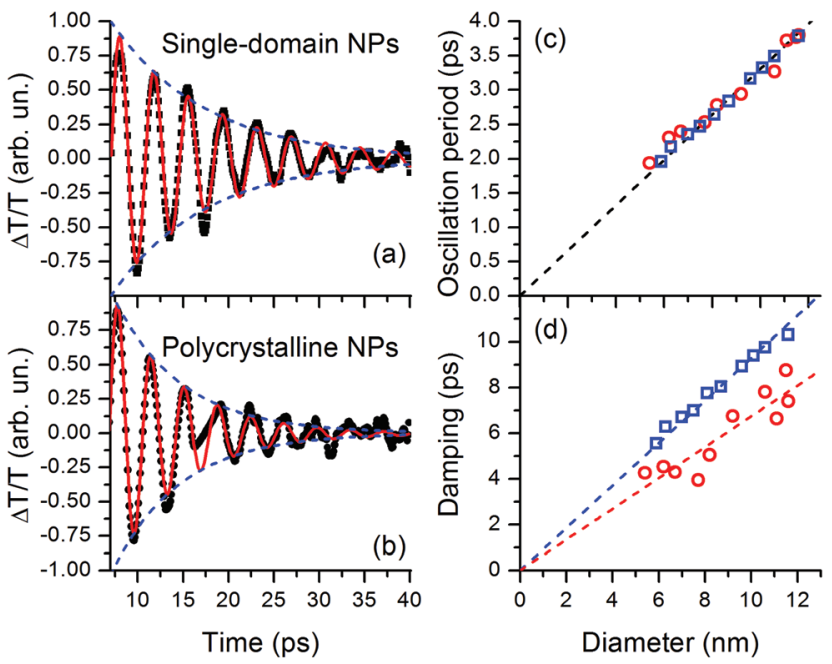

Fig. 7 Normalized oscillations detected at $550 \mathrm{~nm}$ probe wavelength (data points) for single-domain (a) and polycrystalline NPs (b) with $11.6 \mathrm{~nm}$ diameter together with a fit following the model described in the text (red solid curve). Blue dashed lines indicate the envelope of the oscillation amplitude, with $\mathrm{e}^{-t / \tau}$ exponential decay. Period $T$ (c) and damping $\tau$ (d) of the fundamental RBM as a function of the NP diameter $D$ for both single-domain (blue squares) and polycrystalline (red circles) samples, together with linear fits (with fixed zero intercept). For the period (panel (c)) the entire dataset of single-domain and polycrystalline NPs was considered, giving $T=t\left(330 \mathrm{fs} \mathrm{nm}^{-1}\right) D$. For the damping (panel (d)) the two datasets are aligned in lines with different slopes: $\tau_{\mathrm{S}}=(930$ fs $\left.\mathrm{nm}^{-1}\right) D$ for the single-domain NPs (blue squares) and $\tau_{\mathrm{P}}=(670 \mathrm{fs}$ $\left.\mathrm{nm}^{-1}\right) D$ for the polycrystalline ones (red circles).

tation. ${ }^{43,46}$ Following this description, the computed frequencies of the RBM are expected to be almost identical for singledomain and polycrystalline samples ${ }^{25}$ but experimental results in this direction are still controversial. Strong variations were found between single-domain and polycrystalline structures in the case of silver $^{28}$ and hollow gold nanospheres ${ }^{24}$ while no significant effect was observed in $7 \mathrm{~nm} \mathrm{Co}^{22}$ and $5 \mathrm{~nm} \mathrm{Au}$ NPS. $^{23}$

In Fig. 7 panels (a) and (b) we show the RBM oscillations detected at $550 \mathrm{~nm}$ probe wavelength for the $11.6 \mathrm{~nm}$ diameter spherical NPs with different crystallinity (data points). Similar results for all the other NPs are reported in the ESI (Fig. S8 and $59 \dagger$ ). The amplitude of the oscillations rapidly decays in time and after 6-7 periods it vanishes below the sensitivity of our measurements. The decay can be attributed mainly to two mechanisms: (i) intrinsic damping of the RBM caused by energy dissipation, giving rise to a homogeneous broadening and an exponential decay in time $\mathrm{e}^{-t / \tau}$; (ii) dephasing of the oscillations due to inhomogeneous broadening related to the size distribution of the NP, which translates into a superposition of oscillators with different periods, giving rise to a $\mathrm{e}^{-t^{2} / \tau^{2}}$ decay profile. ${ }^{46}$ Both methods provide very good fits for our data (see ESI, Fig. S8 and S9†), with minor differences in the residuals. Anyway, we can exclude a pure inhomogeneous broadening related to the size distribution of the NPs for the following reasons. If the latter was the only cause of the damping of the RBM, the standard deviation $\sigma_{\mathrm{D}}$ of the NP diameter distribution extracted from the pump-probe data would be larger than that estimated from TEM images (see Fig. S2 and S3 in the ESI $\dagger$ ). In fact, knowing the average diameter $\bar{D}$ of each sample, from the fits of the pump-probe data we can extract the oscillation period $T$ and the time constant $\tau$ of the Gaussian decay, so that the standard deviation $\sigma_{\mathrm{D}}$ can be evaluated from the equation ${ }^{46}$ :

$$
\sigma_{\mathrm{D}}=\bar{D} T / \sqrt{2} \pi \tau
$$

The results are plotted in the ESI (Fig. S10(a) $\dagger$ ), together with those obtained from the TEM images, which are an overestimation of the real standard deviation $\sigma_{\mathrm{D}}$ because they are very close to the resolution of the instrument. This allows us to conclude that the major contribution to the damping of the RBM oscillation is the intrinsic damping caused by energy dissipation, thanks to the extremely high purity of the samples (see also the polydispersity extracted from the data in ESI Fig. $\mathrm{S} 10(\mathrm{~b}) \dagger)$. In the following we therefore consider the results obtained from the fits using an exponential decay. The extracted periods of oscillation $T$ and decay time constants $\tau$ are reported in Fig. $7(\mathrm{c})$ and $7(\mathrm{~d})$ as a function of the NP diameter for single-domain (blue squares) and polycrystalline (red circles) NPs. The period of oscillation $T$ increases linearly with the sphere size for both sets of samples. ${ }^{6}$ In accordance with theoretical predictions, ${ }^{25}$ our experiments show that the RBM period is not affected by the nanocrystallinity: the data points in Fig. 7(c) lie on the same straight line. The linear fit of the entire dataset (with intercept set to zero) gives a slope of $\frac{T}{D}=330 \mathrm{fs} \mathrm{nm}^{-1}$, which is in good agreement with the inverse of the longitudinal speed of sound in gold $\frac{1}{v_{\mathrm{s}}}=310 \mathrm{fs} \mathrm{nm}^{-1}$. This suggests that the nanoscale mechanical properties of the NPs, in particular the Young modulus which determines the speed of sound, are not significantly affected by nanocrystallinity. From the analysis of Fig. 7(d) we can conclude that the two datasets lie on straight lines with different slopes: $\frac{\tau_{\mathrm{S}}}{D}=930 \mathrm{fs} \mathrm{nm}^{-1}$ for the single-domain NPs (blue squares) and $\frac{\tau_{\mathrm{P}}}{D}=670 \mathrm{fs} \mathrm{nm}^{-1}$ for the polycrystalline ones (red circles). The quality factors associated with the two crystallinities are then $Q_{\mathrm{S}}=\pi \frac{\tau_{\mathrm{S}}}{T} \approx 9$ for the single-domain and $Q_{\mathrm{P}}=\pi \frac{\tau_{\mathrm{P}}}{T} \approx 6$ for the polycrystalline NPs.

\section{Conclusions}

In this paper we described a novel synthesis method that allows the control of gold NP size and nanocrystallinity using the same chemistry (i.e. nature and concentration of the reagents). We obtained either single-domain or polycrystalline NPs in the size range of 6-13 nm diameter with narrow size distribution. The oxidative etching of oleylamine $/ \mathrm{O}_{2}$ permits the formation of quasi-spherical NPs for both nanocrystalli- 
nities. The availability of such highly controlled samples gave us the unique opportunity to investigate the influence of both the size and nanocrystallinity on the electronic, thermal and mechanical properties of the NPs. We observed that the presence of the twin defects leads to an increase by $20 \%$ in the LSPR linewidth compared to the single-domain NPs. This broadening is attributed to an additional term in the intrinsic electron damping time due to the scattering of electrons on the defects. Unlike plasmons, no influence of nanocrystallinity on electron-phonon equilibration dynamics is observed, suggesting that twins do not significantly enhance the electron-phonon scattering processes. Finally, the frequency and damping time of impulsively excited acoustic vibrations of the NPs are directly probed in the time domain. We show that the elastic properties of the NPs, which determine the speed of sound and thus the vibration period, are not significantly affected by the nanocrystallinity; on the other hand, the damping of the vibrations, through dissipation of the mechanical energy to the environment is sensitive to defects and twins. The damping of the RBM acoustic oscillations can thus be taken as a fingerprint of the nanocrystallinity of the NPs.

\section{Acknowledgements}

Dr Portalès, Dr G. Della Valle and Dr P. Biagioni are kindly acknowledged for fruitful discussions. Dr Albouy is acknowledged for XRD experiments and fruitful discussions. This work was supported by the advanced grant of the European Research Council under no 267129. G.C. acknowledges support by the EC under Graphene Flagship (contract no. CNECT-ICT-604391).

\section{Notes and references}

1 M. H. Huang, S. Rej and S.-C. Hsu, Chem. Commun., 2014, 50, 1634-1644.

2 P. Buffat and J.-P. Borel, Phys. Rev. A, 1976, 13, 2287-2298.

3 G. J. Hutchings and C. J. Kiely, Acc. Chem. Res., 2013, 46, 1759-1772.

4 A. T. Ngo, P. Bonville and M. P. Pileni, J. Appl. Phys., 2001, 89, 3370-3376.

5 C. Salzemann, I. Lisiecki, A. Brioude, J. Urban and M.-P. Pileni, J. Phys. Chem. B, 2004, 108, 13242-13248.

6 G. V. Hartland, Chem. Rev., 2011, 111, 3858-3887.

7 X. Han, Y. Liu and Y. Yin, Nano Lett., 2014, 14, 2466-2470.

8 J. Z. Zhang and C. Noguez, Plasmonics, 2008, 3, 127-150.

9 U. Kreibig and M. Vollmer, Optical Properties of Metal Clusters, Springer, 1995.

10 W. Niu, W. Zhang, S. Firdoz and X. Lu, J. Am. Chem. Soc., 2014, 136, 3010-3012.

11 X. Ye, C. Zheng, J. Chen, Y. Gao and C. B. Murray, Nano Lett., 2013, 13, 765-771.

12 T. Liu, P. Jiang, Q. You and S. Ye, CrystEngComm, 2013, 15, 2350-2353.
13 F. Lu, Y. Zhang, L. Zhang, Y. Zhang, J. X. Wang, R. R. Adzic, E. A. Stach and O. Gang, J. Am. Chem. Soc., 2011, 133, 18074-18077.

14 S. Mourdikoudis and L. M. Liz-Marzán, Chem. Mater., 2013, 25, 1465-1476.

15 M.-P. Pileni, Nat. Mater., 2003, 2, 145-150.

16 N. Zheng, J. Fan and G. D. Stucky, J. Am. Chem. Soc., 2006, 128, 6550-6551.

17 G. Zhou, Y. Yang, S. Han, W. Chen, Y. Fu, C. Zou, L. Zhang and S. Huang, ACS Appl. Mater. Interfaces, 2013, 5, 1334013352.

18 Q. Zhang, J. Xie, Y. Yu, J. Yang and J. Y. Lee, Small, 2010, 6, 523-527.

19 N. Goubet, Y. Ding, M. Brust, Z. L. Wang and M.-P. Pileni, ACS Nano, 2009, 3, 3622-3628.

20 Y.-J. Lee, N. B. Schade, L. Sun, J. A. Fan, D. R. Bae, M. M. Mariscal, G. Lee, F. Capasso, S. Sacanna, V. N. Manoharan and G.-R. Yi, ACS Nano, 2013, 7, 1106411070 .

21 C.-H. Kuo, T.-F. Chiang, L.-J. Chen and M. H. Huang, Langmuir, 2004, 20, 7820-7824.

22 D. Polli, I. Lisiecki, H. Portalès, G. Cerullo and M.-P. Pileni, ACS Nano, 2011, 5, 5785-5791.

23 N. Goubet, C. Yan, D. Polli, H. Portalès, I. Arfaoui, G. Cerullo and M.-P. Pileni, Nano Lett., 2013, 13, 504-508.

24 R. J. Newhouse, H. Wang, J. K. Hensel, D. A. Wheeler, S. Zou and J. Z. Zhang, J. Phys. Chem. Lett., 2011, 2, 228235.

25 A. Crut, P. Maioli, N. D. Fatti and F. Vallée, Phys. Chem. Chem. Phys., 2009, 11, 5882-5888.

26 H. Portales, N. Goubet, L. Saviot, S. Adichtchev, D. B. Murray, A. Mermet, E. Duval and M.-P. Pileni, Proc. Natl. Acad. Sci. U. S. A., 2008, 105, 14784-14789.

27 W. Huang, W. Qian, M. A. El-Sayed, Y. Ding and Z. L. Wang, J. Phys. Chem. C, 2007, 111, 10751-10757.

28 Y. Tang and M. Ouyang, Nat. Mater., 2007, 6, 754-759.

29 J. L. Elechiguerra, J. Reyes-Gasga and M. J. Yacaman, J. Mater. Chem., 2006, 16, 3906-3919.

30 S. Ino and S. Ogawa, J. Phys. Soc. Jpn., 1967, 22, 13651374.

31 G. Wulff, Z. Kristallogr. Mineral., 1901, 34, 449-530.

32 N. W. Ashcroft and N. D. Mermin, Solid State Physics, Harcourt Brace, New York, 1976.

33 C. Novo, D. Gomez, J. Perez-Juste, Z. Zhang, H. Petrova, M. Reismann, P. Mulvaney and G. V. Hartland, Phys. Chem. Chem. Phys., 2006, 8, 3540-3546.

34 C. Sönnichsen, T. Franzl, T. Wilk, G. von Plessen, J. Feldmann, O. Wilson and P. Mulvaney, Phys. Rev. Lett., 2002, 88, 077402.

35 H. Baida, D. Christofilos, P. Maioli, A. Crut, N. D. Fatti and F. Vallée, Eur. Phys. J. D, 2011, 63, 293-299.

36 S. Berciaud, L. Cognet, P. Tamarat and B. Lounis, Nano Lett., 2005, 5, 515-518.

37 O. L. Muskens, G. Bachelier, N. D. Fatti, F. Vallée, A. Brioude, X. Jiang and M.-P. Pileni, J. Phys. Chem. C, 2008, 112, 8917-8921. 
38 C. Voisin, N. Del Fatti, D. Christofilos and F. Vallée, J. Phys. Chem. B, 2001, 105, 2264-2280.

39 G. Della Valle, M. Conforti, S. Longhi, G. Cerullo and D. Brida, Phys. Rev. B: Condens. Matter, 2012, 86, 155139.

40 A. Arbouet, C. Voisin, D. Christofilos, P. Langot, N. D. Fatti, F. Vallée, J. Lermé, G. Celep, E. Cottancin, M. Gaudry, M. Pellarin, M. Broyer, M. Maillard, M. P. Pileni and M. Treguer, Phys. Rev. Lett., 2003, 90, 177401.

41 J. Hodak, I. Martini and G. V. Hartland, Chem. Phys. Lett., 1998, 284, 135-141.

42 B. Dacosta Fernandes, A. Le Beulze, F. Moroté, J. Oberlé, M. Tréguer-Delapierre, J. Burgin and P. Langot, J. Phys. Chem. C, 2013, 117, 22041-22045.

43 N. D. Fatti, C. Voisin, F. Chevy, F. Vallée and C. Flytzanis, J. Chem. Phys., 1999, 110, 11484-11487.

44 K. Yu, J. E. Sader, P. Zijlstra, M. Hong, Q.-H. Xu and M. Orrit, Nano Lett., 2014, 14, 915-922.

45 P. V. Ruijgrok, P. Zijlstra, A. L. Tchebotareva and M. Orrit, Nano Lett., 2012, 12, 1063-1069.

46 G. V. Hartland, J. Chem. Phys., 2002, 116, 8048.

47 J. H. Hodak, A. Henglein and G. V. Hartland, J. Phys. Chem. B, 2000, 104, 9954-9965.

48 G. V. Hartland, Annu. Rev. Phys. Chem., 2006, 57, 403-430.

49 M. F. Cardinal, D. Mongin, A. Crut, P. Maioli, B. RodríguezGonzález, J. Pérez-Juste, L. M. Liz-Marzán, N. Del Fatti and F. Vallée, J. Phys. Chem. Lett., 2012, 3, 613-619.

50 T. A. Major, A. Crut, B. Gao, S. S. Lo, N. D. Fatti, F. Vallée and G. V. Hartland, Phys. Chem. Chem. Phys., 2013, 15, 4169-4176.
51 B. Palpant, H. Portales, L. Saviot, J. Lermé, B. Prével, M. Pellarin, E. Duval, A. Perez and M. Broyer, Phys. Rev. B: Condens. Matter, 1999, 60, 17107-17111.

52 H. Portalès, N. Goubet, S. Sirotkin, E. Duval, A. Mermet, P.-A. Albouy and M.-P. Pileni, Nano Lett., 2012, 12, 52925298.

53 N. Goubet, H. Portalès, C. Yan, I. Arfaoui, P.-A. Albouy, A. Mermet and M.-P. Pileni, J. Am. Chem. Soc., 2012, 134, 3714-3719.

54 D. Polli, L. Lüer and G. Cerullo, Rev. Sci. Instrum., 2007, 78, 103108.

55 S. B. Simonsen, I. Chorkendorff, S. Dahl, M. Skoglundh, J. Sehested and S. Helveg, J. Am. Chem. Soc., 2010, 132, 7968-7975.

56 H. Borchert, E. V. Shevchenko, A. Robert, I. Mekis, A. Kornowski, G. Grübel and H. Weller, Langmuir, 2005, 21, 1931-1936.

57 Y. Zheng, J. Zeng, A. Ruditskiy, M. Liu and Y. Xia, Chem. Mater., 2014, 26, 22-33.

58 J. Lermé, H. Baida, C. Bonnet, M. Broyer, E. Cottancin, A. Crut, P. Maioli, N. Del Fatti, F. Vallée and M. Pellarin, J. Phys. Chem. Lett., 2010, 1, 2922-2928.

59 K.-P. Chen, V. P. Drachev, J. D. Borneman, A. V. Kildishev and V. M. Shalaev, Nano Lett., 2010, 10, 916-922.

60 R. H. M. Groeneveld, R. Sprik and A. Lagendijk, Phys. Rev. B: Condens. Matter, 1992, 45, 5079-5082.

61 H. Lamb, Proc. London Math. Soc., 1881, s1-13, 189-212. 